\title{
Microscopic haematuria associated with increased risk
}

Despite the high prevalence of the disease, patients with bladder cancer often have a poor prognosis because diagnosis relies on a symptomatic patient presenting to their primary-care provider. Symptoms associated with bladder cancer include dysuria, abdominal pain and weight loss, with frank haematuria well established as the highest-risk symptom.

Asymptomatic microscopic haematuria, or nonvisible haematuria, is often detected on routine urine dipstick tests, at a rate of $22 \%$ in men aged $>60$ years. Nonmalignant causes include glomerular disease and UTI, which are more common than a malignant origin. If nonvisible haematuria is detected, the current UK National Institute for Health and Care Excellence (NICE) recommendation for patients aged $<50$ years is nonurgent referral after treatment of potential UTI, or urgent referral in those $>50$ years of age, reflecting the higher prevalence of bladder cancer in this group.

A study recently published in the British Journal of General Practice has shown that

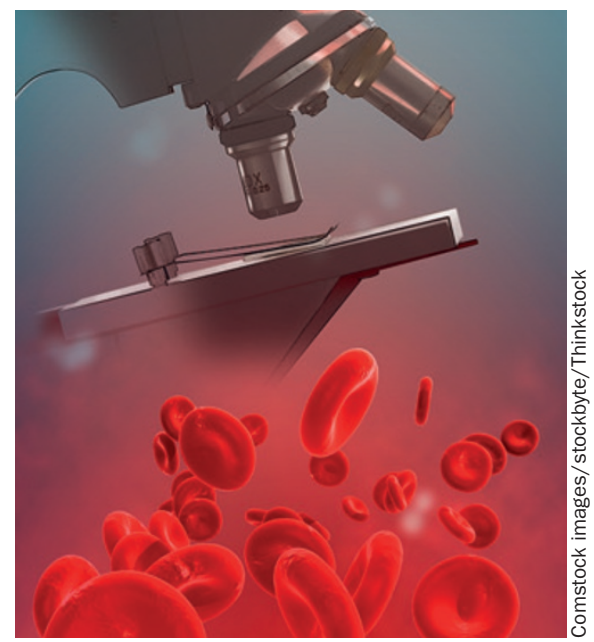

the presence of nonvisible haematuria is indeed a risk marker for bladder cancer, suggesting that further investigation might be more urgently required in older patients. The researchers used an electronic database to match patients with bladder cancer $(n=4,915)$ to controls $(n=21,718)$ by gender, age and general practice; coded and uncoded data were then used to determine the presence of visible or nonvisible haematuria up to 1 year before a cancer diagnosis.

The calculated positive predictive value (PPV) of visible haematuria in this study was $2.8 \%$ in patients aged $>60$ years, and $1.2 \%$ for those $50-59$ years. Nonvisible haematuria was also associated with bladder cancer diagnosis, but with PPVs of around half that of visible haematuria $-1.6 \%$ and $0.8 \%$ in those aged $>60$ years and $40-59$ years, respectively.

This work represents the first study to report an increased risk of bladder cancer in patients in whom nonvisible haematuria is detected; the authors suggest that the NICE guidelines could be amended to include urgent referral of patients aged $>40$ years, as opposed to the current cut-off of 50 years.

\section{Annette Fenner}

Original article Price, S. J. et al. Non-visible and visible haematuria and bladder cancer risk: a study of electronic records in primary care. Br. J. Gen. Pract. 64, e584-e589 (2014) 